Linguistique, littérature, didactique

\title{
Anthropologie et narratologie croisées : à propos des héros de conte folklorique
}

Daniel Aranda

\section{(2) OpenEdition}

Journals

\section{Édition électronique}

URL : http://journals.openedition.org/pratiques/1795

DOI : 10.4000/pratiques.1795

ISSN : 2425-2042

\section{Éditeur}

Centre de recherche sur les médiations (CREM)

\section{Édition imprimée}

Date de publication : 15 décembre 2011

Pagination : 89-100

Référence électronique

Daniel Aranda, «Anthropologie et narratologie croisées : à propos des héros de conte folklorique », Pratiques [En ligne], 151-152 | 2011, mis en ligne le 16 juin 2014, consulté le 19 avril 2019. URL : http:// journals.openedition.org/pratiques/1795; DOI : 10.4000/pratiques.1795

(c) Tous droits réservés 


\title{
Anthropologie et narratologie croisées : à propos des héros de conte folklorique
}

\section{Daniel Aranda}

\author{
Université de Nantes, LabÉCD
}

\section{Introduction}

La question de l'évolution des récits folkloriques est au point mort depuis l'abandon des thèses diffusionnistes développées au XIX siècle, ou des stratégies de reconstitution d'un conte originel et intégral, 1'« Urmärchen » des frères Grimm. Cet échec est préjudiciable à la connaissance d'un genre qui de toute évidence voit ses productions narratives se transformer au fil des générations, mais selon une logique qui nous échappe, et ce en raison du « peu de recul historique dont nous disposons, puisque des collectes systématiques n'ont été entreprises qu'au XIX ${ }^{\mathrm{e}}$ siècle » (Belmont 1999 : 70).

A contrario le conte folklorique semble transparent pour les études narratologiques. En ce qui concerne les personnages par exemple, Philippe Hamon (1984:395) note que « le conte populaire, en revanche, et de façon générale, désigne sans ambiguïté son héros en cumulant sur un même acteur les statuts actantiels de Sujet et de Bénéficiaire ». De fait tout lecteur familier des récits folkloriques sait que la configuration narrative de ses protagonistes est plus complexe ou ambiguë qu'il n'y paraît.

Notre objectif n'est pas de proposer une nouvelle définition du héros. Il est d'améliorer notre compréhension des protagonistes de conte folklorique tels qu'il s'inscrivent concrètement dans les récits, et ce en les confrontant à la théorie du «bouc émissaire » de René Girard. Pour cet auteur l'expression désigne le protagoniste indéfectible des récits mythiques, la représentation essentiellement déformée d'une victime devenue, par le biais de l'histoire mythologique qui la prend en charge, un personnage fabuleux, un monstre sacré au sens propre du terme, qui plonge la communauté dans le chaos mais dont la mise à mort permet à celle-ci de retrouver paix et harmonie :

La conjonction perpétuelle dans les mythes d'une victime très coupable et d'une conclusion simultanément violente et libératrice ne peut s'expliquer que par la force extrême du mécanisme de bouc émissaire. Cette hypothèse, en effet, résout l'énigme fondamentale de toute mythologie : l'ordre absent ou compromis par le bouc émissaire se rétablit ou s'établit par l'entremise de celui qui l'a d'abord troublé [...]. Une victime passe pour responsable des malheurs publics [et] cette même victime ramène l'ordre, le symbolise et même l'incarne. (1982: 64) 
La problématique qui préside à cette comparaison entre le bouc émissaire mythique et le héros de conte folklorique (nous nous limiterons aux récits français, pour l'essentiel merveilleux) associe anthropologie et narratologie. Pour Girard en effet les traits narratifs qui caractérisent le protagoniste du mythe relèvent d'une représentation déformée, déformation qui correspond au fait que l'histoire est rapportée dans la perspective non du bouc émissaire mais de la communauté qui a eu maille à partir avec lui. L'approche est également anthropologique en ce sens qu'un savoir transculturel sur l'être humain - celui que défend notre auteur - prétend déconstruire un autre savoir sur l'être humain, celui que les mythes mettent en œuvre à travers leurs héros. Le héros mythique dans ses caractéristiques narratives résulte d'une perception du personnage qu'une autre interprétation, celle de René Girard, postule comme essentiellement fausse.

Nous adopterons la même démarche pour ce qui est du héros de conte folklorique en nous satisfaisant par hypothèse d'une définition usuelle et générale de ce terme de « héros », celle d'un personnage que des procédés divers mais convergents placent au sommet de la hiérarchie des acteurs de l'histoire, un " conducteur du récit » (Mitterand 1973 : 478) qui assume, pour reprendre le vocabulaire de la sémiologie narrative, le principal rôle actantiel et/ou le principal rôle thématique. Ce personnage, tel qu'il est donné dans les contes populaires, présente un rapport fluctuant mais constant avec le type du bouc émissaire. Ces fluctuations sont intéressantes d'un point de vue actantiel mais aussi d'un point de vue diachronique, en ce sens qu'elles permettent d'envisager une évolution du héros pour ce genre narratif, évolution qui est une proposition de solution au problème que nous avons mentionné précédemment, celui de la difficulté à appréhender diachroniquement les mutations du conte de tradition orale.

La question du conflit des assertions dans le conte folklorique nous permettra d'entrer immédiatement dans le vif du sujet : le narrateur valide ou invalide ce qui est dit du héros par les autres personnages, ce qui nous permet de suivre à la trace une révolution axiologique dans la configuration de ce protagoniste. Or ce lent bouleversement résulte d'un glissement anthropologique, faible mais perceptible, dans la conception du héros de conte qui sera décrit dans un deuxième temps. Enfin cet écart anthropologique permettra d'esquisser une morphogenèse des héros de conte folklorique, c'est-à-dire un scénario qui imagine comment se sont modifiées les qualifications comme le destin de ces personnages.

\section{Véridiction et narration}

Le conflit des assertions, soit l'exposition simultanée d'affirmations incompatibles, est un des ressorts de l'intrigue du conte lorsque est mis en œuvre le motif de l'héroïne accusée à tort d'avoir commis un acte criminel, zoophilie, nécrophagie ou infanticide. À ce motif succède celui de l'héroïne persécutée, le crime qui lui est imputé entraînant systématiquement une coercition : déchéance, mutilation, expulsion ou séquestration. L'examen de ce conflit des assertions permet selon nous de pénétrer la logique d'évolution du récit folklorique merveilleux, fondée sur le renversement de la caractérisation axiologique et actantielle du héros.

\subsection{Deux récits étrangers l'un à l'autre?}

Rapprochons deux contes que tout semble opposer quant à l'histoire. Le premier récit est «Le conte du loup-garou », collecté par Victor Smith le 24 juillet 1874 auprès de Nannette Lévesque à Fraisses-près-Firminy (Loire). Il rapporte que parmi les habitants d'une ferme « la plus jolie des filles se mit loup-garou » (Tenèze-Delarue $2000: 66$ ). Elle dévore les uns après les autres les occupants de l'exploitation jusqu'à ce qu'un jour 
un parent revenu du service militaire vienne rendre visite à la famille. Les voisins lui expliquent que des événements anormaux se produisent dans la maison et il décide d'éclaircir le mystère. Il neutralise la fille loup-garou, appelle le voisinage à la rescousse et la jeune fille est brûlée vive dans le four.

Le second conte s'intitule «Roquelaure, voleur de l'enfant du roi » et fait partie d'une collecte effectuée par Achille Millien dans le Nivernais entre 1885 et 1890. Il comprend deux mouvements qui correspondent à deux générations de héros, la mère puis le fils. Pour se rendre maître d'un enfant aux pouvoirs magiques, le personnage de Roquelaure « écrit au roi que sa femme avait mangé son enfant 》 (Delarue-Tenèze 1977 : 550). La mère est incarcérée, ce qui permet à l'adversaire de disposer de son fils.

Les deux contes proposent un héros féminin qui doit affronter l'adversité sous la forme de personnages antagonistes qui lui reprochent d'avoir commis des crimes identiques : meurtre et manducation de membres de la famille. L'opposition des dénouements tient au fait que dans le « conte du loup-garou » l'héroïne a perpétré ces crimes et est tuée, alors que dans le second elle n'a pas commis ce crime et finit par être réhabilitée. Le changement dans l'organisation des deux histoires est modelé par le bouleversement axiologique du protagoniste : être malfaisant dans la première, innocent dans la seconde.

Or ce renversement est déterminé par la décision du narrateur. En effet le conteur de récits traditionnels hérite d'histoires qu'il transmet mais n'invente pas, sa marge de manœuvre consistant à infléchir l'orientation du récit qu'il rapporte en fonction de multiples critères où figure en bonne place son degré d'adhésion à l'histoire qu'il reçoit. Au fil des siècles et des générations de conteurs, des histoires comparables peuvent se conserver à peu près telles quelles comme se transformer en suivant des directions diverses.

Si donc le conteur valide l'accusation qui pèse sur l'héroïne, celle-ci adopte les allures d'une criminelle dont le châtiment se justifie. Le narrateur aura alors opéré une jonction entre le point de vue des personnages qui accusent le protagoniste et le sien. L'accusation devient la vérité car le narrateur est « digne de confiance par convention » (Rabatel $2008: 24$ ), à telle enseigne que le conte expose que l'héroïne elle même reconnaît les faits. « J'ai tout mangé. Je suis le diable. J'ai fait una pache [un pacte] avec le diable. Je suis le loup-garou » (Tenèze-Delarue 2000 : 71), explique le protagoniste du premier conte. La coïncidence de point de vue entre les différentes instances du récit fait que l'accusation portée contre l'héroïne se confond ontologiquement avec la vérité des faits rapportés.

En revanche, si le conteur opère une disjonction entre la conviction des personnages accusateurs et la présentation des faits dont il assure la régie et l'authentification, le crime imputé à l'héroïne devient une calomnie. Le narrateur se range maintenant du côté du protagoniste à qui il fait adopter la posture d'une victime de la haine ou de l'ambition de ses proches. L'histoire devient celle de sa persécution et de sa réhabilitation. «Roquelaure, voleur de l'enfant du roi » illustre ce choix. Dans ce nouveau conflit d'assertions arbitré par le narrateur, le mensonge calomnieux de l'adversaire entraîne l'adhésion générale des autres personnages de l'histoire mais pas des auditeurs du conte. La conviction des personnages est en porte-à-faux avec celle des auditeurs, avant que le dénouement ne fasse coïncider les deux perceptions. Grâce au conteur, la sphère des auditeurs a basculé du côté de l'héroïne calomniée et injustement persécutée, la sphère des personnages en étant encore, jusqu'au dénouement exclusivement, du côté d'un protagoniste considéré comme coupable et justement châtié.

La matière première de ces deux contes est donc la même, à savoir un conflit aigu entre l'héroïne et son entourage qui porte sur la question de crimes d'une extrême gravité qu'elle aurait perpétrés. Ce qui nous empêche de constater cette identité tient à l'opposi- 
tion des deux récits pour ce qui est de leur habillage axiologique et intrical. Dans un cas le récit s'engage dans l'option narrative d'un être malfaisant qui met en péril la communauté et qu'on parvient à éliminer; dans l'autre elle suit la pente narrative d'une héroïne calomniée et persécutée qui finit par faire éclater la vérité et surmonter l'hostilité de son entourage.

\subsection{D'un conte-type à l'autre}

Pour rendre perceptible l'homologie de ces deux contes, il suffit d'observer dans d'autres récits folkloriques des situations intermédiaires de celles que nous avons examinées. Elles témoignent que l'opposition apparemment binaire entre l'un et l'autre récits correspond en vérité aux deux bornes d'un espace narratif transitionnel qui n'offre pas de solution de continuité. Nous raisonnerons ici en termes de contes-types dans leur adaptation aux contes français (Delarue 1976 et Delarue-Tenèze 1977), et non en termes de versions particulières.

Dans le conte-type ${ }^{\circ} 449$ "L'homme qui a épousé une femme-vampire », le héros se rend compte que sa femme nouvellement épousée se rend la nuit au cimetière pour y dévorer des cadavres. S'ensuit une guerre entre les deux personnages qui se solde par la victoire du héros : la fée n'est pas tuée mais métamorphosée en un animal ou un objet qui assure sa neutralisation et sa déchéance définitives. La différence principale entre cette histoire et celle du « conte du loup-garou » tient dans l'échange des rôles actantiels entre les deux protagonistes : le héros n'est plus la femme-vampire mais celui qui parvient à la réduire à sa merci, le personnage féminin étant désormais l'adversaire. Du coup la table de concordance entre statut actantiel et statut axiologique devient celle des contes folkloriques stéréotypés : le héros est affecté de valeurs positives et l'adversaire de valeurs négatives.

Les contes-types 306 («Les souliers usés à la danse») et 401 («La princesse enchantée libérée après trois nuits d'épreuves ») donnent l'histoire d'un jeune homme qui vient à bout des obstacles que dresse contre lui un adversaire féminin envouté. L'intérêt de ces contes tient aux caractéristiques de la victoire du héros. Le 306 évolue sur une ligne de crête narrative où cohabitent suivant les versions deux dénouements antagonistes : parfois la jeune fille ensorcelée est mise à mort, et nous restons dans la configuration des contes précédents ; parfois l'envoutement est donné comme réversible, ce qui permet à l'adversaire de devenir l'objet de la quête et d'être épousé par le héros, option qui est systématiquement choisie par le type 401.

L'innocence proclamée du personnage dont la monstruosité est désormais réversible lui permet d'obtenir à nouveau le statut d'héroïne. Le type 708 « La fille innocente qui accouche d'un monstre » exploite cette option. Une jeune fille met au monde un enfant contrefait ou un animal, mais la monstruosité de cet acte est indépendante de sa volonté : elle est la victime d'un enchantement opéré par la marâtre et qui vise à la perdre de réputation et la faire condamner. Dès lors son statut de protagoniste est conforme aux récits folkloriques conventionnels.

Que l'ensorcellement se rationalise en complot et nous parvenons au point d'arrivée, celui de « Roquelaure, voleur de l'enfant du roi ». Les contes-types 706 (« La fille aux mains coupées »), 707 ( L'oiseau de vérité »), 713 ( La mère qui ne m'a pas porté, mais m'a nourri ») exploitent le motif de l'héroïne calomniée : un entourage hostile procède à la substitution d'enfants par des animaux lors de l'accouchement de l'épouse, ou dépose un nouveau-né auprès de la jeune fille pour faire croire à une maternité clandestine. Notre abominable fille-loup-garou a donc insensiblement revêtu la physionomie stéréotypée de la princesse persécutée. Et son cheminement est représentatif d'une mutation morphogénétique du conte merveilleux, qui part des histoires à protagonistes mons- 
trueux pour accéder aux histoires à protagonistes persécutés. Si tant de récits nous présentent des héroïnes séquestrées, calomniées, ensorcelées, chassées, c'est qu'ils ont retourné comme un gant l'histoire première, celle d'une héroïne monstrueuse justement châtiée. Le conte folklorique merveilleux comme genre tient dans cette mutation.

\section{Vers une anthropologie du héros de conte folklorique}

\subsection{Récit folklorique et représentation du mécanisme victimaire}

Le conte folklorique merveilleux se déploie dans un espace axiologique et actantiel borné par les récits à héros coupable à une extrémité et par les récits à héros persécuté à l'autre.

La question est de savoir qui dit vrai sur le héros, ou plus exactement quelles sont les proportions de clairvoyance et d'aveuglement dans l'une et l'autre catégories. L'anthropologie religieuse de René Girard servira à établir ce bilan. Cet auteur fournit pour les mythes une interprétation qu'il qualifie d'anthropologique en ce sens qu'elle apporte une explication trans-culturelle de ce genre narratif lui-même trans-culturel. Pour Girard, nous l'avons vu, le mythe rapporte un processus de bouc émissaire dans la perspective faussée et déformante de la communauté qui l'a expérimenté, et qui aboutit à la sacralisation du sujet lynché. Ce mécanisme serait repérable dans tous les mythes non pas parce que l'un aurait influencé l'autre, mais parce qu'il décrit d'une manière toujours déformée à l'identique un processus victimaire universel.

Pour ce qui est du conte, ce processus se repère bien plus dans le « conte du loup-garou » que dans « Roquelaure, voleur de l'enfant du roi ». Ainsi un des stéréotypes du mythe tient aux crimes monstrueux perpétrés par le héros, comme en recèle notre premier récit: "Après qu'il [sic] eut mangé son père, il [sic] alla manger la mère. Il [sic] mangea tout son monde » (Tenèze-Delarue $2000: 68$ ). En revanche le second récit ( « Roquelaure») ne contient pas ce trait. Un deuxième stéréotype tient dans les « signes victimaires » du héros, c'est-à-dire des caractéristiques physiques, mentales ou sociales qui font qu'une collectivité se polarise contre un de ses membres de préférence à un autre. Notre loup-garou cumule quelques traits typiques des sujets les plus vulnérables des communautés traditionnelles, la féminité, la jeunesse, mais aussi cette beauté toujours suspecte : « la plus jolie des filles se mit loup-garou » (ibid. : 66). Le conte « Roquelaure », quant à lui, ne donne aucune indication de ce type. Un troisième stéréotype est la mise à mort collective du personnage monstrueux, qu'illustre également notre premier conte : «ils arrapèrent [sic] la fille et la mirent au four. Quand la fille brûla, la peau jusque là intacte brûla avec elle » (ibid. : 72). Dans « Roquelaure » la sanction existe aussi, mais la mise à mort est remplacée par l'incarcération, transformation indispensable pour que l'histoire se poursuive à l'avantage de la victime.

On peut imaginer que les transformations narratives qui partent du conte à héros monstrueux pour aboutir au conte à héros victime sont la conséquence d'un redressement anthropologique de l'illusion fondatrice véhiculée par les contes qui partagent une même perception du héros que le mythe. Plusieurs observations vont dans ce sens.

D'abord le répertoire français des contes merveilleux compte très peu de contes-types à héros monstrueux ou simplement criminels : le 366 («Rends-moi ma jambe »), le 470A («La vengeance du trépassé »)... Par ailleurs Marie-Louise Tenèze émet des doutes sur le caractère de conte du type $366(2004: 6)$. Enfin pour ce qui est du « conte du loup-garou » recueilli par Victor Smith, s'il est un récit qui se rapproche remarquablement de la description du mythe faite par René Girard, il ne correspond à aucun contetype de la classification internationale Aarne-Thompson. Marie-Louise Tenèze se demande s'il n'est pas la mise en fiction de croyances légendaires au loup-garou encore fortement ancrées, en cette seconde moitié du XIX ${ }^{\mathrm{e}}$ siècle, dans les montagnes du Velay. 
Il se pourrait donc que la conteuse Nannette Lévesque « soit l'auteur de cette transposition, le "créateur de ce conte" » (Tenèze-Delarue 2000: 86). Si c'était le cas, ce récit nous fournirait l'exemple précieux de la naissance d'un conte folklorique à partir d'un substrat légendaire, sans que pour autant nous cherchions à généraliser cette particularité, c'est-à-dire à établir une relation de filiation phylogénétique entre mythe et légende d'un côté, conte de l'autre.

Ainsi le modèle mythique qui nous sert à établir une des frontières de l'espace narratif dans lequel évolue le héros de conte n'existe qu'à l'état résiduel. En revanche, et sans que nous puissions rentrer dans les détails, les proportions entre héros malfaisants et héros innocents s'inversent dans les genres narratifs de la légende ou du mythe. Les mythes sont riches en histoires de héros qui réparent par leur mise à mort ou leur expulsion les catastrophes qu'ils ont déclenchées dans la cité. De même les légendes abondent en créatures monstrueuses qui infestent ou infestaient telle contrée, et dont elles rapportent l'histoire.

À ce paramètre diégétique s'ajoute un paramètre pragmatique. Les événements représentés sont supposés être archétypalement vrais dans le mythe ou factuellement vrais dans les légendes. Pour le conte, en revanche, le mode de consommation est fictionnel : conteurs et auditeurs s'entendent sur le fait que l'histoire rapportée ne doit pas faire l'objet d'une croyance. Cette différence entraîne une conséquence à la fois technique et culturelle. Technique : légende et surtout mythe exigent un souci de fidélité de la part du conteur qui ne doit rien y mettre du sien, puisqu'il y va de la préservation de la vérité qu'ils véhiculent. Le régime fictionnel lève en revanche cet interdit et donne davantage de latitude au conteur. La vitesse d'infléchissement des contes serait donc supérieure à celle des mythes et légendes. Culturelle : si, comme l'affirme Jean-Marie Schaeffer avec Hume, « il faut penser la fiction non par rapport à la vérité mais par rapport à la croyance » (2004: 165), le conte apprend à l'auditoire à prendre ses distances vis-à-vis de l'acte de foi qu'exigent de lui la légende et plus encore le mythe. Le récit folklorique serait alors le promoteur d'un espace partiellement émancipé de cette parole intangible qui régit les communautés archaïques, et qui s'en protège justement par sa nature fictionnelle, insignifiante a priori. Nicole Belmont note ainsi (1999: 63) que « dans la société traditionnelle, les contes ne firent jamais l'objet d'interdits de la part des autorités religieuses, contrairement aux croyances et aux rituels populaires ».

\subsection{Une mutation anthropologique faible}

Par rapport aux mythes et aux légendes, la spécificité anthropologique du conte folklorique tiendrait donc dans un effort perceptible pour s'émanciper de la logique du héros monstre sacré. Le protagoniste de conte est soit celui qui répare les méfaits que subit une communauté clanique ou familiale, soit beaucoup plus rarement celui qui engendre ces méfaits ; mais il n'est jamais comme le héros mythique celui qui les produit pour mieux les réparer, encore moins celui qui devient une divinité à laquelle sont attachés comme des emblèmes ce mal et ce remède. Pour ce qui est du renversement axiologique et intrical qui s'effectue à l'intérieur de l'espace du conte, les observations que nous avons faites dans les sections précédentes accréditent l'hypothèse que le conte fait preuve d'une plus grande pénétration vis-à-vis du phénomène de bouc émissaire qu'il rapporte. Le retournement du héros coupable en héros victime traduirait une révolution anthropologique, celle de la mise à jour de l'innocence du bouc émissaire, accusé à tort dans la mesure où étant orphelin, ou mineur, ou étranger au clan, etc., il est incapable de déclencher des représailles. Cependant un examen plus poussé des contes les plus «évolués » nous fait penser que la réalité est plus complexe qu'il n'y paraît.

Une observation triviale le montre : dès qu'un conte déculpabilise une héroïne de type « conte du loup-garou » en en faisant une héroïne innocente de type «Roquelaure», 
il génère immédiatement et systématiquement un ou des personnages exécrables de rechange. Toujours absents des contes à héroïne monstrueuse, les marâtres, sorcières, sœurs ou demi-sœurs haineuses et autres personnages du même acabit surgissent immanquablement dans les récits de la variété « Roquelaure ». Ils sont des victimes de rechange de l'héroïne, des boucs émissaires au second degré auxquels est attribué le même pouvoir néfaste, fait de sorcellerie et d'intelligence perverse mêlées. Leur physionomie comme leur destin reproduisent exactement ceux des héroïnes coupables : monstruosité et lynchage. Même si des récits de la variété " conte du loup-garou » n'étaient pas parvenus jusqu'à nous, il aurait été possible de reconstituer le prototype du héros monstrueux en considérant les adversaires de l'héroïne persécutée dont ils sont le modèle réduit. Dans le second conte, Roquelaure est le même personnage qui accuse la reine d'avoir tué et mangé son fils et qui, une fois en possession de l'enfant, ordonne à sa servante de le tuer et de le préparer pour un repas anthropophage : « tu me le feras cuire pour mon goûter» (Delarue-Tenèze 1977 : 550).

À ce titre, l'aveuglement anthropologique des contes à héroïne innocente est semblable à celui des contes à héroïne monstrueuse. Des structures mentales homologues sont à l'œuvre, qui ne peuvent imaginer que la négativité du monde ne soit imputable à un sujet particulier ou une communauté particulière, et que leur anéantissement ne suffise pour redonner à la société son lustre d'antan. Mais la situation est tellement enchevêtrée qu'on peut encore apporter une réserve à ce qui se présentait déjà comme une restriction à notre hypothèse de départ. Par rapport aux légendes et aux mythes, le conte folklorique a ceci de particulier qu'il promeut la notion largement inédite jusqu'ici d'innocence persécutée, et dans un même mouvement s'intéresse plus aux victimes qu'aux coupables. Il fait du spectacle de cette persécution surmontée son morceau de bravoure, alors que le châtiment des adversaires, s'il est maintenu dans la plupart des cas, est expédié en une phrase au dénouement. Non seulement l'innocence existe, mais elle devient plus intéressante que la culpabilité. Au final l'évolution du conte enregistrerait une mutation anthropologique faible, celle-ci entraînant une mutation narrative forte.

\section{Narratologie morphogénétique du héros de conte folklorique}

Esquissons un scénario morphogénétique qui rende compte des configurations successives du héros dans le conte folklorique. Ce jeu de mutations s'inscrit dans une zone de transition entre deux postures anthropologiques voisines, suffisamment distinctes cependant pour que le passage de l'une à l'autre enregistre une révolution dans la considération axiologique et actantielle du héros.

\subsection{Du monologisme au dialogisme}

L'hypothèse qui est expérimentée dans cette étude veut que les contes à héroïne monstrueuse engendrent les contes à héroïne persécutée, et non l'inverse. Elle s'appuie sur la différence de statut discursif entre ces deux catégories de récits. Le second groupe relève du discours dialogique (au sens bakhtinien du terme) puisque deux histoires, rapportant le même épisode mais ne s'entendant pas sur les événements qui s'y sont produits, existent simultanément dans chaque conte. En effet un micro-récit à héroïne monstrueuse est systématiquement enchâssé dans tout récit à héroïne persécutée. Des récits momifiés et enkystés comme celui-ci sont légion :

La belle-mère prit la lettre qu'il [le courrier] portait et la remplaça par une autre qu'elle écrivit où il était dit au prince que sa femme avait accouché d'un chien et d'une chienne, on y demandait ce qu'il fallait faire de ces animaux et de leur mère. (Sadoul $1904: 559)$ 
Certes dans ces récits les adversaires ne croient pas à la vérité des propos qu'ils tiennent, à la différence des faits rapportés dans un récit comme «Le conte du loup-garou ». Mais les personnages auxquels ils s'adressent y croient et en tirent les conclusions. Pour son entourage fictionnel l'accouchement monstrueux rapporté par l'adversaire a le même degré d'évidence que le cannibalisme de la fille loup-garou pour les auditeurs du « conte du loup-garou».

En revanche il n'y a jamais de discours défenseurs instillés dans les contes à protagoniste monstrueux. Quand ceux-ci font parler l'hérö̈ne, c'est pour la faire adhérer à ce discours unanime qui crée la réalité en la rapportant. La fille loup-garou reconnaît les faits et supplie qu'on la mette à mort : «Tuez-moi, si vous ne me tuez pas, j'en mangerai bien d'autres » (Tenèze-Delarue 2000 : 71). Les contes à héroïne monstrueuse sont donc monologiques en ceci qu'ils proposent une unification intégrale des faits représentés. Si l'on adopte le présupposé selon lequel l'évolution interne des récits de tradition orale va du simple au complexe et non l'inverse, cette disparité conforte notre hypothèse. Les micro-récits accusateurs produits par les adversaires sont des résidus de contes à héroïne coupable dans lesquels se sont enracinés et développés des contes à héroïne calomniée. Ils sont des vestiges recyclés de l'histoire initiale, dont la nouvelle fonction est de faire triompher provisoirement le mensonge sur la vérité, c'est-à-dire de promouvoir une péripétie dans l'histoire.

\subsection{Fortunes de l'héroïne monstrueuse}

L'héroïne monstrueuse engendre trois catégories de protagonistes, ceux à culpabilité affirmée, ceux à culpabilité atténuée, ceux à culpabilité niée. Bien que les deux derniers résultent du premier par bifurcation, chacune de ces trois figures propose un scénario plus abouti que le précédent pour s'éloigner de la configuration de l'héroïne prototypique.

Si le héros à culpabilité affirmée n'existe qu'en nombre restreint dans le répertoire des contes merveilleux, c'est qu'il apparaît comme aberrant dans la posture idéologique dominante du genre, qui veut que le statut de héros soit associé à des qualifications et actions positives. L'hésitation dans les dénouements attribués à ces histoires traduit le malaise du conteur face à cette configuration : le héros meurt ou au contraire sort victorieux des épreuves selon les versions d'un même conte-type. Retenons pour cette catégorie de protagonistes, bien que les deux dernières histoires appartiennent au registre des « contes religieux », trois contes-types qui prennent pour argent comptant les méfaits de l'héroïne tout en les actualisant en mensonge (type 710), stérilité volontaire (type 755), orgueil (type 756 A). L'histoire d'une héroïne monstrueuse devient à peu de frais l'histoire édifiante d'une pécheresse que des pénitences parfois atroces, qui recyclent de fait les sévices infligés au protagoniste malfaisant, permettront ou non de sauver de l'enfer.

Mais le plus souvent le maintien de la culpabilité du protagoniste entraîne sa migration actantielle, et à ce titre ne concerne plus directement notre propos. La fonction de héros n'est donc qu'un des rôles possibles du personnage du bouc émissaire intervenant dans le conte folklorique. Pour ce genre narratif, le destin du héros malfaisant est de ne pouvoir rester héros, ou de ne pouvoir rester malfaisant.

Cette seconde option connaît une expérimentation avec le héros à culpabilité atténuée. Les crimes de l'héroïne primordiale demeurent, mais leur édulcoration radicale permet au personnage de rester le protagoniste. Les fameuses désobéissances bénignes, maladresses, curiosités déplacées ou malchance persistante qui caractérisent tant de héros de contes merveilleux provoquent des catastrophes qui constituent le moteur de l'intrigue. Le protagoniste va devoir rétablir l'équilibre qu'il a rompu par une série d'épreu- 
ves. La transformation du crime en bévue, celle de la mise à mort en épreuve, engendrent une matrice de scénarios stéréotypés dont la productivité est considérable et connue de tous.

Le passage de la culpabilité atténuée à la culpabilité niée parachève la moralisation du héros, celui-ci transférant intégralement ses caractéristiques malfaisantes aux adversaires et faux héros. Il ne reste de sa monstruosité qu'une présomption fallacieuse, selon un couple d'assertions contradictoires que nous avons analysé précédemment et qui constitue le nouveau moteur de l'intrigue. Le type de l'héroïne persécutée avec calomnie engendrera une forme ultime, celle de l'héroïne persécutée sans calomnie, à l'instar de Cendrillon (type 510A). Les adversaires ne cherchent plus à manipuler l'opinion et se prévalent de leur seul pouvoir face à la vulnérabilité de la victime, parce que même le spectre mensonger d'une monstruosité de l'héroïne apparaît au conteur comme inconvenant, vis-à-vis par exemple d'un auditoire enfantin. L'effacement des traces devient alors total.

L'héroïne calomniée connaît parfois un changement de statut actantiel à l'intérieur d'une même histoire. Une fois séquestrée, elle transmet à ses enfants le rôle de héros. Les contes-types 652 («Le prince dont tous les souhaits se réalisent»), 707 («L'oiseau de vérité ») et 708 ( « La fille innocente qui accouche d'un monstre ») proposent des histoires en deux temps, chacun ayant son héros propre, la mère calomniée pour la première, le fils vengeur pour la seconde, la mère endossant dans ce dernier cas le rôle d'objet de la quête effectuée par ses enfants. Le temps des héros quêteurs est venu.

\subsection{Du héros victime au héros quêteur}

La distinction entre " héros victime » et « héros quêteur » revient à Vladimir Propp (1970 [1928] : 48). Elle est d'autant plus pertinente que selon nous ces deux rôles ne correspondent pas au même personnage de départ. Le passage de la victime au quêteur se fait par changement de personnage, alors que la mutation du héros coupable en héros innocent résulte de l'évolution d'un personnage unique. L'antique monstre sacré cède son poste de héros à un autre intervenant dans cette histoire primitive de lynchage, un membre de cette foule effervescente focalisée contre sa victime.

Si l'acteur victime est celui que la communauté désire éliminer pour que cesse la crise dont il serait le promoteur, l'acteur quêteur est celui qui résout cette même crise en annihilant le personnage nuisible. La difficulté est qu'il ne saurait y avoir de combat moralement justifié contre le héros dès lors que celui-ci en vient à être présenté comme une victime persécutée. Du coup l'action de coercition punitive va muter en action de sauvetage. Pour reprendre le «conte du loup-garou » qui nous sert de référence, l'enjeu de l'histoire sera toujours moins : " comment le visiteur va-t-il venir à bout de la fille loupgarou ?» et sera toujours plus : «comment va-t-il pouvoir la sauver, et même l'épouser?»

Deux jalons témoignent de cette montée en puissance d'un des agents de la mise à mort de la victime. Le militaire qui intervient dans la seconde partie du « conte du loupgarou » n'est pas le héros de l'histoire mais l'adversaire, adversaire qualifié positivement et victorieux. Le dédoublement du héros monstrueux en une victime ensorcelée (ou captive, etc.) et un personnage nuisible qui la tient à sa merci, permet à l'adversaire de devenir adjuvant à travers deux tâches complémentaires, l'une punitive comme à l'origine (châtier le personnage malfaisant) et l'autre désormais salvatrice, libérer la princesse séquestrée par le personnage malfaisant. Les contes-types 510A («Cendrillon »), 511 («Un-œil, Double-œil, Triple-œil»), 709 (« Blanche-Neige ») mettent en scène un tel adjuvant, qui prend d'autant plus de volume qu'il occupe également le poste d'« objet » pour l'héroïne qui l'épouse au dénouement. 
Une version angoumoise de l'histoire de la Belle au bois dormant (type 410) donne une indication sur la manière dont notre adjuvant-objet a pu accéder au statut de héros. L'incipit est en effet le suivant : «Un jour, le fils du roi, étant à la chasse avec ses invités, s'égara dans la forêt en poursuivant le cerf» (Massignon 2006 [1983] : 207). Le récit débute donc in medias res pour ce qui est des mésaventures de la jeune fille, les événements antérieurs étant rapportés au fils du roi (et donc aux auditeurs) par la princesse enchantée ou sa marraine. Cette disjonction entre l'ordre du récit et celui de l'histoire va de pair avec l'échange des postes actantiels entre personnages : le héros, celui dont on suit les aventures depuis le début de l'histoire, est maintenant le prince, et non la Belle qui n'intervient qu'en cours de route et au titre d'objet de la quête du héros.

Il suffit donc que le conteur mette plus en avant le personnage qui sauve par rapport à celui qui est sauvé pour franchir un seuil actantiel et ouvrir la voie à tous ces contes-types - 300, 313, 408, 531... - qui mettent en scène un héros quêteur dont la mission consistera à délivrer une jeune fille. Lorsque Marie-Louise Tenèze note (2004 : 10) que « la Malfaisance, dans l'opposition externe s'exerce sur l'Objet, alors que dans l'opposition interne elle s'exerce sur le Héros », elle repère une bifurcation narrative qui rejoint notre hypothèse génétique : il y a "malfaisance » dans les deux cas parce que celle-ci s'exerce toujours sur le même personnage. Simplement dans l'une des options narratives il est héros alors que dans la seconde il est devenu objet, laissant la place à un hérosquêteur.

\section{Conclusion}

Notre hypothèse et l'option méthodologique qui en découle présentent l'intérêt de placer anthropologie et narratologie dans un rapport d'interaction. D'une part elles établissent la pertinence de la conception ethno-anthropologique de René Girard lorsqu'elle est appliquée au conte folklorique; d'autre part elles permettent de mieux rendre compte des héros de récits populaires merveilleux, dans leurs manifestations atypiques comme dans leurs configurations les plus stéréotypées. Comme le mythe ou la légende, le conte folklorique ne cesse de rapporter l'histoire d'un bouc émissaire, avec cette particularité (qui a valeur de signature) que de lourdes mutations axiologiques et diégétiques l'ont rendu le plus souvent méconnaissable. Cependant une approche comparative - rapprocher des versions d'un même conte-type ou des contes-types entre eux - peut reconstituer ces métamorphoses avec un degré de probabilité non négligeable.

Rassemblons les principaux résultats de cette étude à travers l'évocation d'un contetype « d'animaux », le $\mathrm{n}^{\circ} 212$, intitulé « La chèvre menteuse ». Le conflit des assertions s'y retrouve : dans une version de l'Albret («La petite chevrette ») un père envoie l'un après l'autre ses sept enfants, puis sa femme, son chien, son chat, emmener paître sa chèvre. Chacun prend soin de lui faire manger tout son soûl, mais au retour la chèvre affirme à chaque fois qu'il n'en a rien été : «Le chevrier d'aujourd'hui ne m'a pas bien gardée ! De trois mesures de lait que j'avais je n'en ai plus qu'une cuillerée » (Dardy 1986 [1891] : 414). Chaque soir, à cette nouvelle, le père pris de colère coupe la tête du chevrier du jour et la jette dans un puits. Lorsqu'il ne reste plus que lui pour mener la chèvre, celle-ci lui tient le même discours et le père se rend compte de sa méprise. Ainsi la vérité des faits finit par être partagée par toutes les instances présentes dans l'histoire, père compris, et cette vérité stigmatise la chèvre puisque ses mensonges à répétition provoquent la destruction violente de toute une famille. Les caractéristiques du personnage (animal versus être humain, et en dernière instance animal domestique versus animal familier) traduisent à la fois une altérité vis-à-vis des chevriers et une proximité insinuante qui va de pair avec sa duplicité. Le châtiment de tels forfaits va de soi : comme nos jeunes filles monstrueuses ou prétendues telles, la chèvre est blessée (Dardy 1986 
[1891] : 418), dépecée vivante (Smith 2005 : 32), décapitée (Arnaudin 2001 [1887]: 572), démembrée et jetée dans un puits (Grandpré 1903 : 369).

Les différentes versions du conte-type «La chèvre menteuse » traduisent de même un processus de transformation du statut axiologique du personnage de monstre en victime. Même dans les récits les plus accusateurs (ainsi de la version Dardy) la colère meurtrière du père dédouane dans une certaine mesure la responsabilité de l'animal. Mais les récits les plus significatifs établissent que la chèvre ne ment pas toujours, ou même pas du tout, et ce grâce à des procédés parfois ingénieux, le plus souvent laborieux. Telle version nous présente un animal qui ment la première fois mais pas les suivantes, car les frères survivants l'empêchent réellement de se repaître :

- T'as fait tuer mon frère,

J'vas t'attacher,

Que tu ne pourras pas manger. (Ibid.)

Telle autre version nous explique qu'effectivement « le petit garçon ne lui donnait pas le temps de brouter» (Sébillot $1900: 424$ ). L'attribution de l'acte fatal se déplace donc de la chèvre aux chevriers - ce sont eux qui mentent désormais - par un phénomène de bouc émissaire du second degré que nous avons déjà rencontré. Une autre version rapporte que la chèvre dit vrai quand elle répond au chevrier qu'elle est « bien saoule », et encore vrai quand elle répond au père qu'elle n'est « ni saoule, ni rassasiée », car dans l'intervalle de ces deux affirmations elle a tout déféqué, au point qu'« elle n'a plus rien dans le ventre » (Tenèze $1976: 208$ ). Une autre version accrédite les propos de la chèvre en imaginant qu'« elle avait un trou au cou de sorte que ce qu'elle mangeait, du moins la plus grande partie, sortait par ce trou » (Smith $2005: 31$ )... Ici la résistance de la forme du conte au travail de réfection inspiré par le renversement axiologique du personnage est flagrante. L'essentiel est que la chèvre menteuse ne mente plus vraiment. Du coup elle peut passer du statut d'adversaire à celui d'héroïne positivement qualifiée. D'assez nombreuses versions collectées accordent la vie sauve à la chèvre pour lui permettre de prolonger l'histoire, en tant que protagoniste, avec le conte-type 123 « Le loup, la chèvre et ses chevreaux ».

Comme le montre le conte-type «La chèvre menteuse », le récit folklorique évolue dans un espace anthropologique presque homogène - il faut toujours un coupable mais l'écart qui est mis à jour suffit pour faire effectuer au personnage une révolution axiologique. À son tour l'axiologie intervient comme facteur d'accélération ou de résistance aux transformations actantielles du héros et à l'évolution concomitante de ses qualifications. Concrètement, le héros victime du conte populaire se présente comme une mutation de la figure archaïque du bouc émissaire, que l'on trouve dans bien des mythes et des légendes mais aussi, à l'état résiduel, dans quelques contes qui présentent des protagonistes monstrueux. En revanche le héros-quêteur est une mutation non du bouc émissaire mais de son adversaire, une version individuée de la foule rassemblée contre lui, à l'instar du personnage du père dans «La chèvre menteuse » qui fait encore partie de la communauté en crise mais s'en détache par l'autorité et le pouvoir de sanction qui lui sont conférés.

Le bouc émissaire passe d'un poste actantiel à un autre - celui de héros notamment ce passage entraînant pour lui beaucoup ou peu de modifications internes. Parallèlement le héros peut ou pas être qualifié positivement, peut ou pas réussir dans son entreprise. Un scénario morphogénétique permet d'imaginer comment ont pu se succéder ces diverses positions actantielles et ces caractéristiques contradictoires. Il en ressort que les fonctions de héros, objet, adjuvant, adversaire, etc., des descriptions formalistes ou structuralistes ne sont pas des essences, mais de simples stases qui ont fixé plus ou moins longtemps tel ou tel personnage, à travers tel conte-type notamment, mais qui 
peuvent se résorber comme se renforcer en fonction de l'évolution de l'économie interne de l'histoire. Cependant cette labilité ne doit nullement être considérée comme le résultat d'une combinatoire gratuite, d'une série de coups de dés dans on ne sait quelle littérature potentielle. En tant que bouc émissaire, c'est un personnage de monstre sacré déjà formé, déjà armé d'un destin et de qualifications spécifiques, qui se fraye un passage à travers les possibles narratifs du conte, et les transformations qu'il expérimente lors de son périple phylogénétique dépendent autant des structures mentales des conteurs qui le prennent en charge que des contraintes propres aux voies dans lesquelles il s'engage.

\section{Références bibliographiques}

ARNAUDIn, F. (2001 [1887]) [conteur : F. Labrit] : « La chèvre», Contes populaires de la Grande-Lande, Mont-de-Marsan, éd. Confluences, 572-577.

Belmont, N. (1999) : Poétique du conte, Paris, Gallimard.

DARDY, L. (1986 [1891]) : "La petite Chevrette », Anthologie populaire de l'Albret, t. 2, Béthaire, éd. Navéra, 414-427.

Delarue, P. (1976) : Le Conte populaire français, t. 1, Paris, Maisonneuve et Larose.

Delarue, P., \& TenĖze, M.-L. (1977) : « Roquelaure, voleur de l'enfant du roi », Le Conte populaire français, t. 2, Paris, Maisonneuve et Larose, 549-552.

GIRARD, R. (1982) : Le Bouc émissaire, Paris, Grasset.

GRANDPRÉ, M.-E. de (1903), «La chèvre et les sept gars », Revue des Traditions Populaires, 18, 369-370.

HAMON, Ph. (1984) : «Héros, Héraut, Hiérarchies », in Le Personnage en question, Toulouse, Service des Publications de l'université Toulouse-Le Mirail, 387-398.

MASsignON, G. (2006 [1983]) [conteur : M. Ganachaud] : « La Belle au bois dormant », De bouche à oreille, Paris, José Corti, 207-208.

MitTerand, H. (1973) : «Fonction narrative et fonction mimétique », Poétique, 16, 477 490.

PROPP, Vl. (1970 [1928]) : Morphologie du conte, trad. M. Derrida, T. Todorov, C. Kahn, Paris, Seuil.

RABATEL, A. (2008) : Homo narrans, 2 vol., Limoges, Lambert-Lucas.

SAdOUL, Ch. (1904) [conteur : Cl. Hüssler] : «La fille aux mains coupées ou l'hôtesse du Dragon vert », Revue des Traditions Populaires, 19, 557-562.

SCHAEFFER, J.-M. (2004) : «Fiction et croyance », in N. Heinich \& J.-M. Schaeffer, Art, création, fiction, Nîmes, éditions Jacqueline Chambon, 163-186.

SÉBILlot, G. (1900) [conteuse : E. Melon] : «La chèvre », Revue des Traditions Populaires, 8, 424-425.

Smith, V. (2005) [conteuse : Sœur Sainte Claire] : «L'homme aux trois chèvres », Contes $d u$ Velay, commentaires de M.-L. Tenèze, Retournac, Société des amis du musée de Retournac, 31-33.

TENÈZE, M.-L. (1976) : « La bête Malibête », Le Conte populaire français, t. 3, Paris, Maisonneuve et Larose, 208-211.

- (2004) : Les Contes merveilleux français, Paris, Maisonneuve et Larose.

TENÈZE, M.-L., \& Delarue, G. (2000) : «Le conte du loup-garou », Nannette Lévesque, conteuse et chanteuse, Paris, Gallimard, 66-72. 